\section{Discussion : Acquisition and processing by computer of chemical laboratory data}

\section{A. E. RAPPOPORT}

The mission of a clinical laboratory is to perform tests accurately, swiftly, and economically. The constantly growing demand for laboratory studies is matched by a critical paucity of adequately trained technologists necessary to perform them. Laboratories are too small and cramped to accommodate present technical staffs and equipment and costs are sky rocketing.

As a result of these developments, we have experienced over the past decade the introduction into the laboratory of newly invented mechanized devices which have taken over or eliminated many of the hitherto manual acts involved in test performance. In order better to measure the impact of this technological revolution, it is necessary to analyse the typical automated laboratory test into its major components and subject each to a qualitative and quantitative re-evaluation as a result of these recent technological developments.

An automated test comprises at least two major parts: (1) the collection of an appropriate specimen and physical performance of the scientific procedure which eventually leads to the establishment of a measurable reaction or result: (2) the execution of a large amount of paper work necessary to document the entire procedure.

The chief features of the mechanical portion of the test are: phlebotomy; centrifugation; automaticintroduction of a given amount of specimen into the system by aspiration, pumping, or pipetting; discrete movement or continuous flow of the sample through the various stations of the device; dispensing reagents; heating; moving the treated specimen into a reaction chamber; automatic removal of waste byproducts; creation of a final colour reaction; automatic entry of the sample into a measuring device (spectrophotometer) possessing a flow-through cuvette; generation of an electrical signal which represents either absorbance or percentage $T$. This is a measurement but not the final result.

Documentation includes all of the paper work which precedes and follows the above briefly described process. This activity includes identification of the patient, specimen, and test to be performed; creation of appropriate calibration curves or reference standard values; establishment of 'normal' values for quality control procedures (standard deviation, coefficient of variation, cusum); transferring these results to the patient's laboratory report; delivery of report to the proper patient in the correct nursing station for the attention of an identifiable attending physician.

All of these steps must be carried out rapidly in order to create a report to be of maximum value in the treatment of the patient. There are many variations to this scheme which one may encounter in different tests, in different laboratories operating under different modes.

Previous speakers in this symposium have discussed many of the technical advances in test performance and have presented excellent reviews of continuous flow and discrete instruments and combinations of these. Some of these automated devices have been on the market for approximately 10 to 12 years and have established their role in the laboratory world and, to a large extent, have eliminated much of the manual effort.

On the other hand, until a short time ago, significant improvement in laboratory documentation capability was not demonstrable. However, it is now possible to report on the recent introduction into laboratories of another automated system, namely data processing, whose function is to process the information involved in test documentation as described above. These two discrete, automated systems can therefore be merged into a single, continuous process which extends the range of automation from specimen procurement and test performance through calculation of the result to its subsequent entry into the computer and finally the preparation of a consolidated updated, printed laboratory report presented to the physician.

A third automated system has recently made its appearance in the laboratory and, acting as a bridge, is being joined to the other two automatic techniques. Teleprocessing devices permit automatic acquisition of the patient's demographic information from the originating nursing station and transmit these requests to the laboratory. The final reports are also returned to the nursing stations by these same teleprocessing devices which have the ability to create hard (printed) copy or to display the results visually by cathode ray tube terminals. These may be combined with typewriter consoles which permit inquiry concerning data into and response from a distant computer.

Thus, it is possible now to consider the possibility of a shared laboratory system in which close proximity of laboratory to the patient need not be entirely necessary.

We have created at the Youngstown Hospital a centralized, cybernetic, clinical laboratory information and data acquisition system in which numerous automated and semi-automated laboratory instruments were successfully connected to an IBM 1080 data acquisition system and an IBM 360 model 30 computer to achieve specimen identification, documentation of test performance, and to prepare totally computerized patients' laboratory reports for the attending physican.

Specimens from one unit are delivered several times daily to the other unit where they join similar samples originating at that unit, and all are tested in large batches. The two unit laboratories (North Unit and South Unit) have been joined into a unified communications network using teleprocessing (IBM 2780, 1050) devices.

The completely automated procedures performed daily at the North Unit include 21 AutoAnalyzer chemical tests and four AutoAnalyzer (SMA-4A) haematology tests. In this system, patients and specimens are automatically identified by prepunched, numbered stub cards attached to the Vacutainer specimen tubes. These are read automatically by the IBM 1084 and 1894 sampler readers with the Technical sampler 40 reader and thus are accessed 
into the 1081 processing unit. The test result peak voltages are obtained from the strip chart recorders and also entered into the system, where they are digitized through A/D converters. All these data are finally input into the computer where, by appropriate programs, the final result values are calculated and stored in the patients' files in the computer.

Semi-automated procedures are those usually performed on non-mechanized, electronic, stand-alone, instruments such as the $p \mathrm{H}$ meter, spectrophotometer, fibrometer, densitometer, and flame photometer which, through specially constructed electronic interfaces, have been connected to the IBM 1080 system. Although the actual test performance is semi-automated, demographic data such as the patient identification and all test documentation including calibration, calculation, and preparation of the report are completely computerized. Pencil and paper have been virtually eliminated in the cybernetics system and personal effort has been markedly reduced.

Manual examinations such as urine analyses, differential blood smears, pathological anatomy (surgical pathology, necropsy, and histology), virology, cytogenetics, exfoliative cytology, and certain special complex chemical and microbiological procedures are also centralized at the North Unit, while all blood banking and serological procedures for syphilis are performed at the South Unit. Facilities for the manual performance of most necessary or 'stat' procedures are available at both units as back-up in the event of failure in either the testperforming or data-processing functions. All clerical and secretarial effort, including key punching, has also been transferred to the North Unit.

The results of all manually performed examinations are key-punched in the conventional manner or entered into Port-A-Punch cards at the work bench. These are transmitted by teleprocessing terminals (IBM 2780, 1050-1052) to the computer at the North Unit. Microbiology and pathology narrative reports and pathologists' consultations are typed into the computer on this terminal. Thus, all laboratory results are collected in each patient's record in the random disc files of the computer.

A ward report is prepared at $1.00 \mathrm{pm}$ to report the current morning's results. Updated, consolidated patient summary reports are printed daily in the evening and include all results obtained that week from Sunday through Saturday, thus permitting horizontal chronological review and comparison. These are sent to the wards at both North and South Units for insertion into the patients' clinical records. Since the computer is located at the North Unit, it prints reports there for North Unit patients, while all reports for South Unit patients are simultaneously printed at that unit by the printer of the IBM-2780.

It must be pointed out that this is a shared, off-line, batch system, in which about two and a half hours of the $360 / 30$ are devoted to laboratory data acquisition and information systems. Simultaneously with the professional activities, such acts as tallying of tests, quality control reviews, and charging of patients, are being carried out.

It should be obvious that this cybernetic method of laboratory organization permits the attainment of the few simple goals postulated in the opening paragraph of this paper.

Intensive studies are under way to assess the 'cost effectiveness' of these efforts in terms of achieving maximum utilization of personnel, space, and equipment at a reduced cost and with enhanced reliability, accuracy, and reproducibility.

Modern laboratory medicine must meet the challenges of the present with the technology available today. 